\title{
A better Approach to detect the Presence of Cell Phones being used in Prohibited Areas
}

\author{
Pankaj Mangal \\ VIT University \\ Vellore.
}

\author{
Shubham Kumar \\ VIT University \\ Vellore
}

\author{
Rajkumar S. \\ VIT University \\ Vellore
}

\begin{abstract}
This handy, pocket-size mobile transmission detector can sense the presence of an activated mobile phone from a distance of one and half meters. So it can be used to prevent the use of mobile phones in examination halls, confidential rooms, etc ${ }^{[2]}$. There are existing models for the cell phone detectors which works when some transmission occurs from the phone. In this paper, we are proposing a detector which works even when the mobile phone is just in the switched on mode but not being used for any kind of transmission. It is also useful for detecting the use of mobile phone for Spying and unauthorized video transmission ${ }^{[2]}$. The circuit can detect the incoming calls and outgoing calls, SMS and video transmission even if the mobile phone is kept in the silent mode. The moment the bug detects RF(Radio Frequency) transmission signal from an activated mobile phone, it starts sounding a beep alarm and LED blinks ${ }^{[1]}$. The alarm continues until the signal transmission ceases.
\end{abstract}

\section{General Terms}

General terms we are going to use here is cell phone detector, EM waves(RF), Security ${ }^{[2]}$, privacy in restricted and confidential areas et. al.

\section{Keywords}

Cell phone detector; No transmission mobile detector; RF(Radio Frequency) detector; Exam malpractice detector; security; privacy; confidential

\section{INTRODUCTION}

In the modern world of high competition, there are many exams being conducted daily. The students of this new technology world are becoming more and more intelligent in doing malpractices in the exams. In the maximum cases, the students are found to use blue tooth devices connected to their mobile phones and following the malpractices. For the prevention of these wrong practices, presently the detectors being used can only identify the presence of a mobile phone when, the mobile is in use, or there is some kind of transmission occurring from the phone.

Mobile phone uses RF with wavelength of $30 \mathrm{~cm}$ at 872 to $2170 \mathrm{MHz}^{[1]}$. That is the signal with high frequency and energy. When the mobile phone is active, it transmits the signal in the form of sin waves which pass through the space. The encoded audio/video signal contains EM wave signal which is picked up by the receiver in the base station. As soon as EM wave is detected by the receiver, the circuit gets activated. The transmitter power of $2 \mathrm{G}$ antenna in the base station is 20-100 Watts. The mobile phone transmits short signals at regular intervals to register its availability to its nearest base station. Distance to a cellular base station is the most important environmental factor ${ }^{[1]}$. Generally the nearer cell phone is to a base station or transmitting tower, the weaker will be the signal that needs to come from the cell phone.

It detects the incoming and outgoing calls, SMS, video transmission even when the phone is kept in silent mode, since illegal use of cell phones is a growing and dangerous problem in correctional institutions worldwide ${ }^{[1]}$.

Cerpa et.al ${ }^{[1]}$. Proposed a tiered system architecture of data collection after various filters. It proposes a mechanism which detects the outliers in filtered data. In order to verify the results, Habitat monitoring database is selected. Author claims through results that the periodic observations of the system detect the outliers in an efficient way. However, this system is silent on the performance issues of the individual sensor or the network. This system may be used in many practical areas as $^{[2]}$ :

$\checkmark \quad$ Preventing the use of mobile phones in combustible delivery stations and petrol pumps.

$\checkmark \quad$ Preventing the use of cell phones in highly confidential areas like Military related secured zones, Court of laws, Historical places, hospitals and Embassies.

$\checkmark \quad$ Preventing the use of mobile phones in important conference halls and exam halls and for spying and unauthorized video transmission.

\section{BASIC CONCEPT AND WORKING OF CELLPHONE DETECTOR}

This circuit is intended to detect unauthorized use of mobile phones in examination halls, confidential rooms etc. It also helps to detect unauthorized video and audio recordings ${ }^{[2]}$. It detects the signal from mobile phones even if it is kept in the silent mode. It also detects SMS

\subsection{Concept}

Mobile phone uses RF with a wavelength of $30 \mathrm{~cm}$ at 872 to $2170 \mathrm{MHz}$. That is the signal is high frequency with huge energy. When the mobile phone is active, it transmits the signal in the form of sine wave which passes through the space $^{[4]}$. The encoded audio/video signal contains electromagnetic radiation which is picked up by the receiver in the base station. Mobile phone system is referred to as "Cellular Telephone system" because the coverage area is divided into "cells" each of which has a base station ${ }^{[4]}$. The transmitter power of the modern $2 \mathrm{G}$ antenna in the base station is 20-100 watts ${ }^{[4]}$.

When a GSM (Global System of Mobile communication) digital phone is transmitting, the signal is time shared with 7 other users. That is at any one second, each of the 8 users on the same frequency is allotted $1 / 8$ of the time and the signal is reconstituted by the receiver to form the speech. Peak power output of a mobile phone corresponds to 2 watts with an 
average of 250 milli watts of continuous power. Each handset with in a 'cell' is allotted a particular frequency for its use. The mobile phone transmits short signals at regular intervals to register its availability to the nearest base station.

The network data base stores the information transmitted by the mobile phone. If the mobile phone moves from one cell to another, it will keep the connection with the base station having strongest transmission ${ }^{[4]}$. Mobile phone always tries to make connection with the available base station. That is why, the back light of the phone turns on intermittently while traveling. This will cause severe battery drain. So in long journeys, battery will flat within a few hours ${ }^{[8]}$.

AM Radio uses frequencies between $180 \mathrm{kHz}$ and $1.6 \mathrm{MHz}$, FM radio uses 88 to $180 \mathrm{MHz}$, TV uses 470 to $854 \mathrm{MHz}^{[6]}$. Waves at higher frequencies but within the RF region is called Micro waves ${ }^{[4]}$. Mobile phone uses high frequency RF wave in the micro wave region carrying huge amount of electromagnetic energy ${ }^{[6]}$. That is why burning sensation develops in the ear if the mobile is used for a long period. Just like a micro wave oven, mobile phone is 'cooking' the tissues in the ear. RF radiation from the phone causes oscillation of polar molecules like water in the tissues ${ }^{[7]}$. This generates heat through friction just like the principle of microwave oven. The strongest radiation from the mobile phone is about 2 watts which can make connection with a base station located 2 to 3 km away.

\subsection{How the circuit works?}

Ordinary LC (Coil-Capacitor) circuits are used to detect low frequency radiation in the AM and FM bands. The tuned tank circuit having a coil and a variable capacitor retrieve the signal from the carrier wave. But such LC circuits cannot detect high frequency waves near the microwave region. Hence in the circuit, a capacitor is used to detect RF from mobile phone considering that, a capacitor can store energy even from an outside source and oscillate like LC circuit.

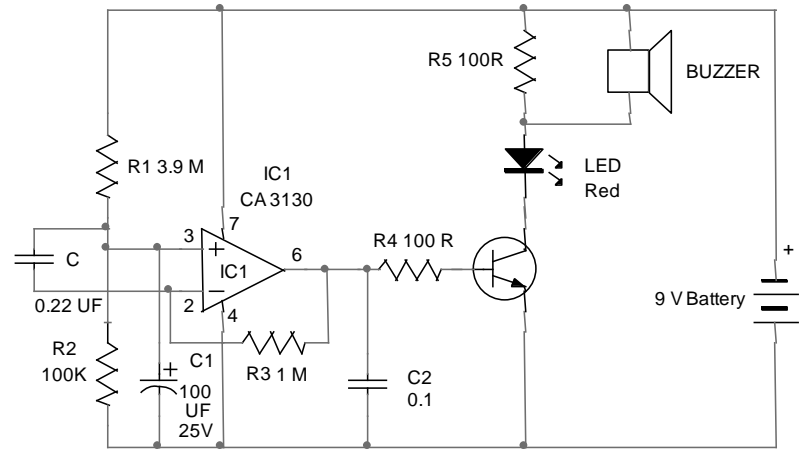

Fig. 1: Working of the circuit

\subsection{Use of capacitor.}

A capacitor has two electrodes separated by a 'dielectric' like paper, mica etc. The non polarized disc capacitor is used to pass AC and not DC. Capacitor can store energy and pass AC signals during discharge. $0.22 \mu \mathrm{F}$ capacitor is selected because it is a low value one and has large surface area to accept energy from the mobile radiation ${ }^{[1]}$.

To detect the signal, the sensor part should be like an aerial. So the capacitor is arranged as a mini loop aerial (similar to the dipole antenna used in TV).In short with this arrangement, the capacitor works like an air core coil with ability to oscillate and discharge current.

\subsection{How the capacitor senses RF?}

One lead of the capacitor gets DC from the positive rail and the other lead goes to the negative input of IC1. So the capacitor gets energy for storage. This energy is applied to the inputs of IC1 so that the inputs of IC are almost balanced with 1.4 volts. In this state output is zero ${ }^{[1]}$. But at any time IC can give a high output if a small current is induced to its inputs. There a natural electromagnetic field around the capacitor caused by the $50 \mathrm{~Hz}$ from electrical wiring.

When the mobile phone radiates high energy pulsations, capacitor oscillates and release energy in the inputs of IC. This oscillation is indicated by the flashing of the LED and beeping of Buzzer. In short, capacitor carries energy and is in an electromagnetic field. So a slight change in field caused by the RF from phone will disturb the field and forces the capacitor to release energy ${ }^{[3]}$.

\section{PROPOSED METHOD}

This model works even when the mobile is just in the switched on mode but not being used for any kind of transmission. The block diagram of the proposed model is shown below ${ }^{[9]}$ :

i) The Radio Frequency signal emitted from the phone is sensed by the antenna and it makes to transmit some current to the IC 3130

ii) IC 3130 then converts the input small current released by the 0.22 capacitor as output voltage.

iii) Any increase in voltage at + input will change the output state to high. Normally IC 3130 is off. So IC 555 will be also off. When the power is switched on, as stated above, IC 3130 will give a high output and Transistor conducts to trigger LED and Buzzer

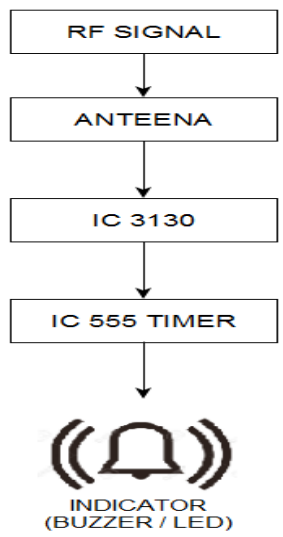

Fig. 2: flow chart for demonstration.

\subsection{Overview of mobile bug:}

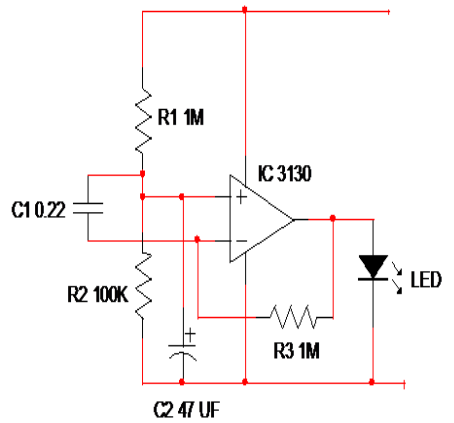

Fig. 3: overview to the circuit. 
$>$ IC1 is designed as a differential amplifier non inverting input is connected to the potential divider $\mathrm{R} 1, \mathrm{R} 2$.

$>$ IC1 functions as a current to voltage converter, since it converts the tiny current released by the 0.22 capacitor as output voltage.

$>$ At power on output go high and LED lights for a short period. This is because + input gets more voltage than the - input. After a few seconds, output goes low because the output current passes to the - input through R2. Meanwhile, capacitor $\mathrm{C} 1$ also charges.

$>$ So that both the inputs gets almost equal voltage and the output remains low. 0.22 capacitor (no other capacitor can be substituted) remains fully charged in the standby state.

> When the high frequency radiation from the mobile phone is sensed by the circuit, 0.22 cap discharges its stored current to the + input of IC1 and its output goes high momentarily. (in the standby state, output of the differential amplifier is low since both inputs get equal voltage of 0.5 volts or more).

$>$ Any increase in voltage at + input will change the output state to high. Normally IC1 is off. So IC2 will be also off. When the power is switched on, as stated above, IC1 will give a high output and T1 conducts to trigger LED and Buzzer .This can be a good indication for the working of the circuit.

\subsection{Circuit Diagram}

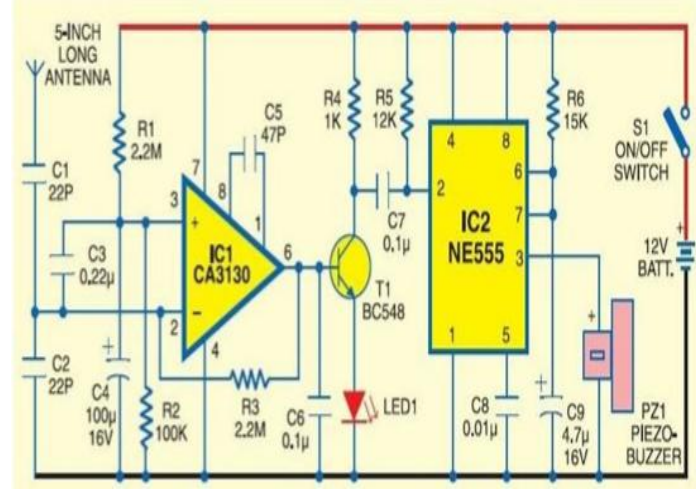

Fig. 4: circuit diagram to implement a simple mobile sensor.

The above circuit diagram represents that the introduced model consists of two IC, few capacitors and resistors.

\section{HARDWARE IMPLEMENTATION}

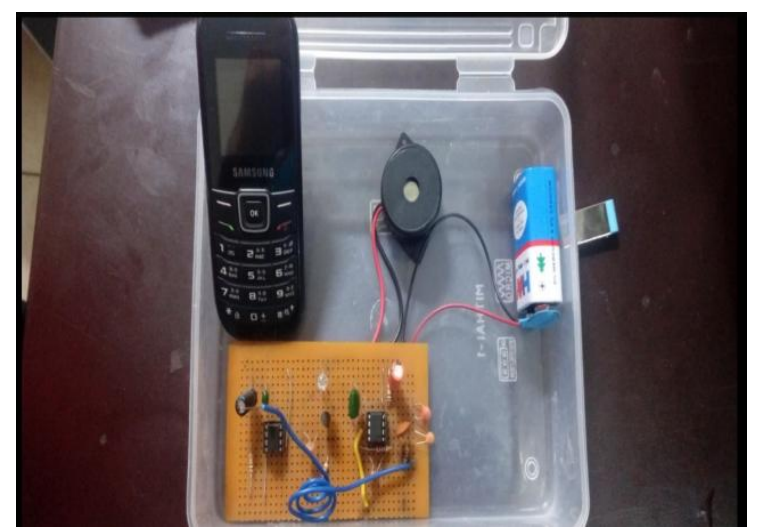

Fig. 5: The hardware implementation.

\section{ACKNOWLEDGMENTS}

The authors take this occasion to thank God, almighty for blessing them with his grace and taking their endeavour to a successful Culmination. They extend their sincere thanks to the Professors of VIT university for providing them with the right guidance and advice at the crucial junctures and for showing them the right path.

\section{CONCLUSION}

The introduced model does have a bright future scope since it is the demand of the emerging technology. The technology has provided many fast gadgets which are being used for multipurpose malpractice and against the prohibition.

\section{REFERENCES}

[1] Philipp Baumann 1, Reynold J. Cooper2 , Dorit S. Hochbaum 1, Nidhi Patel2, Kunal Shalia 11 "Efficient Deployment of Mobile Detectors for Security Applications” ,Department of Industrial Engineering and Operations Research, University of California, Berkeley, USA 2Lawrence Berkeley National Laboratory, Berkeley, USA

[2] T. Carpenter, J. Cheng, F. Roberts, and M. Xie, "Sensor management problems of nuclear detection," in Safety and Risk Modeling and Its Applications, H. Pham, Ed. London: Springer Series in Reliability Engineering, 2011.

[3] V. A. Traag, A. Browet, F. Calabrese and F. Morlet, "Social Event Detection in Massive Mobile Phone Data Using Probabilistic Location Interference", SocialCom/PASSAT, pp.625-628, October 9-11,2011

[4] J. W. Branch, C. Giannelia, B. Szymanski, R.Wolff and H. Kargupta, "In-Network Outlier Detection in Wireless Sensor Networks", knowledge and information systems, vol 31, Springer-Verlag, 2012.

[5] M. M. Breunig, H.P. Kriegel, R.T. Ng and J. Sander, "LOF: Identifying Density Based Local Outliers," In Proceedings of the ACM SIGMOD Conference, Dallas, TX, May 2000

[6] B. Wang and W. Perrizo, "RDF: a density based outlier detection method using vertical data representation", IEEE Int. Conference on Data Mining, pp. 503-506, 2004.

[7] S. Rajagopalan, R. Karwoski, B. Bartholmai, R. Robb, "Quantitative image analytics for strtified pulmonary medicine", IEEE Int. Symposium on Biomedical Imaging (ISBI), pp. 1779-1782, 2012.

[8] V.chandola, A.Banerjee and V .kumar, "Outlier Detection:A survey", ACM Computing surveys,pp. 172,2009.

[9] J. Yang and Y. wang, "A new outliers detection algorithms based on Markov chain", Advance Materials Research, vol;:366, pp.456-459, 2012.

[10] D.M. Hawkins, "Identification of outliers", Chapman and Hall,London, 1980. 